Check for updates

Cite this: RSC Adv., 2017, 7, 25622

\title{
Method for electroless nickel plating on the surface of $\mathrm{CaCO}_{3}$ powders
}

\begin{abstract}
Junjun Huang (D) acde and Zhenming Chen ${ }^{\star a b}$
In this work, electroless nickel plating on the surface of $\mathrm{CaCO}_{3}$ powders successively modified with stearic acid, oleamide and 3-amino-propyltriethoxysilane in that order was developed. Hydrophobic and hydrophilic coatings were deposited onto the surface of the $\mathrm{CaCO}_{3}$ powders in that order. On the one hand, the hydrophobic chains coated onto the surface of the modified powders could effectively protect the powders from chemical reaction with acidic solutions (the sensitizing solution and the activating solution). On the other hand, active groups formed on the surface of the hydrophilic coating could adsorb a palladium catalyst for electroless nickel plating. It was observed that $\mathrm{Ni}-\mathrm{P}$ particles were deposited on the surface of the modified $\mathrm{CaCO}_{3}$ powders after electroless plating. The size of the particles was less than $100 \mathrm{~nm}$. The mechanical bonds between the modified powders and the plated particles were strong, leading to good adhesion. The saturation magnetization of the plated powders was around $1.5 \mathrm{emu} \mathrm{g}^{-1}$. By changing the type of inorganic powder and plating bath, the established method can potentially be adopted for the plating of metals such as copper, cobalt, and nickel onto different types of powders.
\end{abstract}

Received 16th March 2017

Accepted 24th April 2017

DOI: 10.1039/c7ra03110f

rsc.li/rsc-advances
It is well known that the sensitizing solution and activating solution are usually acidic $\mathrm{SnCl}_{2}$ solution and acidic $\mathrm{PdCl}_{2}$ solution, respectively, where the $\mathrm{pH}$ is adjusted using $\mathrm{HCl}$ so as to avoid the formation of $\mathrm{Sn}(\mathrm{OH}) \mathrm{Cl}$ and $\mathrm{Pd}(\mathrm{OH})_{2},{ }^{11-13}$ which would reduce the catalytic performance. This will facilitate the formation of $\mathrm{Sn}^{2+}$ and $\left[\mathrm{PdCl}_{4}\right]^{2-}$ in the acidic solutions. Only the $\mathrm{Sn}^{2+}$ and $\left[\mathrm{PdCl}_{4}\right]^{2-}$ ions in the sensitizing solution and activating solution are useful for electroless reactions. ${ }^{\mathbf{1 1 - 1 3}}$ However, many carbonate powders (such as $\mathrm{CaCO}_{3}$ and $\mathrm{MgCO}_{3}$ ) easily react with acids, so these powders cannot be activated in acidic $\mathrm{PdCl}_{2}$ solution.

Calcifying organisms incorporate carbon directly from seawater into their skeletons in the form of inorganic minerals such as $\mathrm{CaCO}_{3}$, and many species of invertebrate have shells or skeletons made of $\mathrm{CaCO}_{3}$. Calcite or marble is crushed and graded to produce many kinds of technical natural $\mathrm{CaCO}_{3}$ with different fineness. ${ }^{14} \mathrm{CaCO}_{3}$ powders are characterized by low cost, high whiteness and natural abundance. ${ }^{14}$ They are widely used in rubber, plastic, paint, and paper. Nickel-plated $\mathrm{CaCO}_{3}$ powders may help to expand the applications of conductive fillers and electromagnetic shields. Polymer-based composites will have good anti-static and electromagnetic shielding properties if a resin is added into the plated $\mathrm{CaCO}_{3}$ to reduce the resistivity. In addition, plated $\mathrm{CaCO}_{3}$ has wide applications in electronic slurries, conductive paints and other fields because of its good oxidation resistance, thermal stability and low cost. However, activation of $\mathrm{CaCO}_{3}$ in acidic $\mathrm{PdCl}_{2}$ solution and then electroless nickel plating onto the surface of $\mathrm{CaCO}_{3}$ is still not well studied. In this work, electroless nickel plating onto the 
surface of $\mathrm{CaCO}_{3}$ powders successively modified with stearic acid, oleamide and 3-amino-propyltriethoxysilane (KH550) in that order was developed. The structural properties and magnetic performance of the plated powders were investigated systematically using an LCR bridge, scanning electron microscopy (SEM) and X-ray diffraction (XRD).

\section{Experimental}

Commercial $\mathrm{CaCO}_{3}$ powders were purchased from Kelong Micro-powder Co. Ltd (Hezhou, China). The size of the particles was 800 mesh. The powders were dropped into a high-speed mill at $453 \mathrm{~K}$ for $30 \mathrm{~min}$. Then, the high-speed mill was cooled to $373 \mathrm{~K}$. Stearic acid (0.2 wt\%) and oleamide $(0.8 \mathrm{wt} \%)$ were added twice at intervals of $30 \mathrm{~min}$ in order, and stirring of the mixture was continued for another $20 \mathrm{~min}$. After that, the covered powders were immersed in KH550 solution (40 wt\%) at $60{ }^{\circ} \mathrm{C}$ for $5 \mathrm{~min}$, and then rinsed again and dried in an oven at $80{ }^{\circ} \mathrm{C}$ for $60 \mathrm{~min}$. The modified $\mathrm{CaCO}_{3}$ powders were sensitized with $\mathrm{SnCl}_{2}$ solution (adjusted to $\mathrm{pH} 1$ using $\mathrm{HCl}$ ) and activated with $\mathrm{PdCl}_{2}$ solution (adjusted to $\mathrm{pH} 2$ using $\mathrm{HCl}$ ). Then the powders were cleaned with deionized water. After that, the powders were immersed in an electroless nickel plating bath $\left(\mathrm{Na}_{3} \mathrm{C}_{6} \mathrm{H}_{5} \mathrm{O}_{7} \cdot 2 \mathrm{H}_{2} \mathrm{O} 8 \mathrm{~g} \mathrm{~L}^{-1}, \mathrm{NiSO}_{4} \cdot 7 \mathrm{H}_{2} \mathrm{O} 5 \mathrm{~g} \mathrm{~L}^{-1}, \mathrm{NH}_{4} \mathrm{Cl} 18 \mathrm{~g} \mathrm{~L}^{-1}\right.$, $\mathrm{NaH}_{2} \mathrm{PO}_{2} \cdot \mathrm{H}_{2} \mathrm{O} 15 \mathrm{~g} \mathrm{~L}^{-1}$ ). The bath temperature was $333 \mathrm{~K}$. The $\mathrm{pH}$ value was 10 . The plating time was $3 \mathrm{~min}$. Then, the plated powders were ultrasonically treated for $20 \mathrm{~min}$.

The surface morphology of the powders was observed by SEM (JEOL, JSM-5600LV). The chemical structures of the samples were measured by XRD (Rigaku D/max-2550V) and X-ray photoelectron spectroscopy (XPS, Shimadzu, AXIS Ultra DLD). Information on the surface functionalities of the powders was obtained by Fourier transform infrared spectroscopy (FT-IR, Thermo, Nicolet 6700). A links systems energy dispersive spectrometer (EDS) was used for elemental analysis. The magnetic performance of the plated powders was investigated using an LCR bridge (JEOL, HP4284A).

\section{Results and discussion}

Fig. 1 shows the FT-IR spectra of the pristine $\mathrm{CaCO}_{3}$ powders (a), and the powders modified with stearic acid and oleamide (b), and then KH550 (c) in that order. The peaks at about $876 \mathrm{~cm}^{-1}$, $1040 \mathrm{~cm}^{-1}, 1430 \mathrm{~cm}^{-1}, 1454 \mathrm{~cm}^{-1}$ and $\sim 3000 \mathrm{~cm}^{-1}$ are due to the $\left(\mathrm{C}-\mathrm{O}_{3}\right)^{2-}, \mathrm{C}-\mathrm{N}, \mathrm{C}-\mathrm{O}, \mathrm{N}-\mathrm{H}_{2}$ and $\mathrm{O}-\mathrm{H}$ characteristic peaks, respectively. ${ }^{11,15,16}$ As shown in Fig. 1(b), the peak at $\sim 3000 \mathrm{~cm}^{-1}$ disappeared and a peak at $1454 \mathrm{~cm}^{-1}$ appeared. ${ }^{11,15,16}$ These results indicated that amine and hydrophobic groups were attached onto the surface of the $\mathrm{CaCO}_{3}$ powders modified with stearic acid and oleamide. The hydrophobic chains could effectively protect the $\mathrm{CaCO}_{3}$ powders from moisture and ion penetration. As shown in Fig. 1(c), the relative intensity of the peak at $1454 \mathrm{~cm}^{-1}$ increased and a peak at $\sim 3000 \mathrm{~cm}^{-1}$ appeared. These results indicated that more amine groups were attached onto the surface of the powders. In addition, the surface of the powders became hydrophilic, which is helpful for

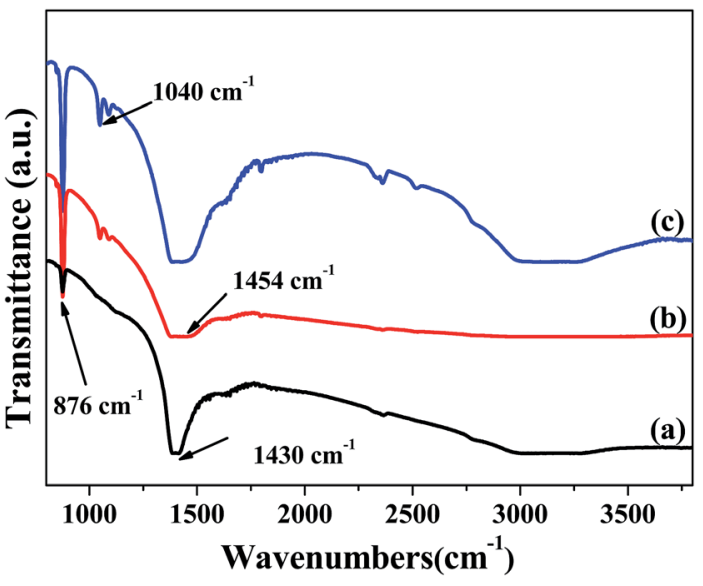

Fig. 1 FT-IR spectra of (a) the pristine $\mathrm{CaCO}_{3}$ powders, and the powders modified with (b) stearic acid and oleamide, and then (c) KH550 in that order.

the dispersion of the modified powders in the sensitizing solution, activating solution and plating solution.

Fig. 2 shows the XPS spectra of the (a) pristine $\mathrm{CaCO}_{3}$ powders, (b) modified powders, (c) sensitized powders, (d) activated powders and (e) plated powders. The peaks at about $286 \mathrm{eV}, 336.3 \mathrm{eV}, 398.8 \mathrm{eV}, 439 \mathrm{eV}, 486 \mathrm{eV}, 534 \mathrm{eV}, 649 \mathrm{eV}, 728 \mathrm{eV}$ and $795 \mathrm{eV}$ are due to C 1s, Ca 2p3/2, Pd 3d, N 1s, Ca 2s, Sn 3d, O 1s, Ni, and Sn 3p, respectively. ${ }^{17,18}$ The peak positions of Ca $2 \mathrm{p} 3 /$ 2 and Pd $3 d$ are very close. As shown in Fig. 2(b), the characteristic peak of nitrogen was detected, which indicated that amine groups were formed on the surface of the modified $\mathrm{CaCO}_{3}$ powders. Meanwhile, an Sn 3d signal was detected in the spectrum, as shown in Fig. 2(c). This indicated that the surface structure of the modified powders facilitated the adsorption of tin ions. Fig. 3 shows the deconvoluted Pd 3d XPS spectra of the (a) modified $\mathrm{CaCO}_{3}$ powders, (b) sensitized powders and (c) activated powders. The characteristic peak of palladium was only detected in the XPS spectrum of the activated powders. As shown in Fig. 3(c), the peak at about $336.3 \mathrm{eV}$ was assigned to Pd $3 \mathrm{~d}$, which is below the standard value of the $\mathrm{Pd} 3 \mathrm{~d}$ peak position

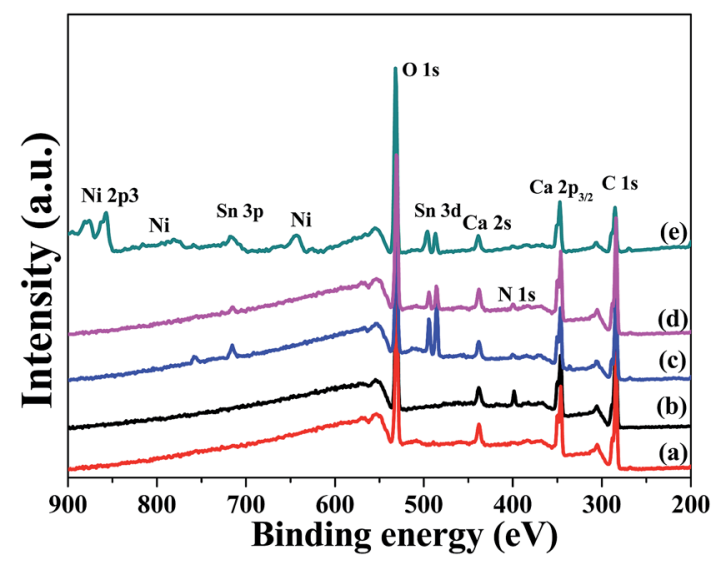

Fig. 2 XPS spectra of the (a) pristine $\mathrm{CaCO}_{3}$ powders, (b) modified powders, (c) sensitized powders, (d) activated powders and (e) plated powders. 


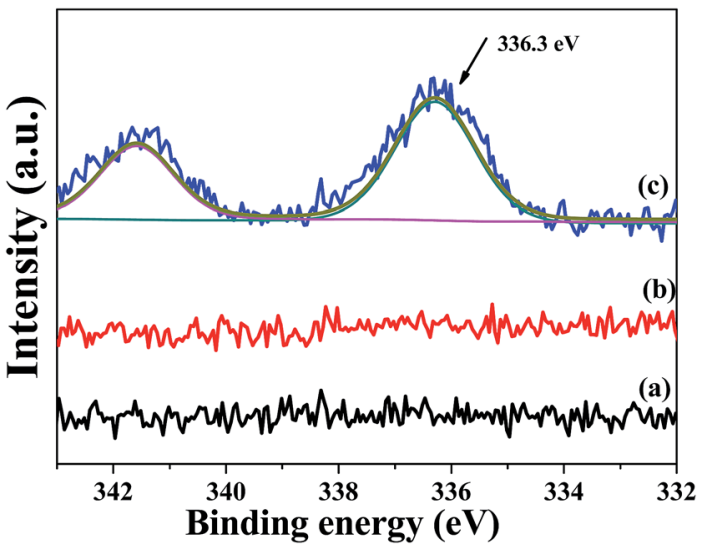

Fig. $3 \mathrm{Pd}$ XPS spectra of the (a) surface modified $\mathrm{CaCO}_{3}$ powders, (b) sensitized powders and (c) activated powders.

$(338.4 \mathrm{eV}){ }^{19,20}$ These results indicated that the active groups adsorbed Pd atoms via chelation, which reduces the electron density around the Pd atoms. ${ }^{19-21}$ It was indicated that the surface structure of the modified powders facilitated the chemisorption of palladium ions. The chemisorbed Pd atoms were used as the catalyst for electroless plating, ${ }^{\mathbf{8}, 18,19}$ and as shown in Fig. 2(e), an Ni signal was detected in the spectrum. The chemical reaction process can be summarized as follows: ${ }^{15}$

$$
\mathrm{Ni}_{(\mathrm{aq})}{ }^{2+}+\mathrm{H}_{2} \mathrm{PO}_{2(\mathrm{aq})}{ }^{-}+\mathrm{H}_{2} \mathrm{O}_{(\mathrm{l})} \stackrel{\text { catalyst+heat }}{\longrightarrow} \mathrm{H}_{(\mathrm{aq})}{ }^{+}+\mathrm{HPO}_{3(\mathrm{aq})}{ }^{2-}+\mathrm{Ni}_{(\mathrm{s})}
$$

Sodium hypophosphite cannot reduce $\mathrm{Ni}^{2+}$ directly. The Pd atoms can adsorb sodium $\mathrm{H}_{2} \mathrm{PO}_{2}{ }^{-}, \mathrm{H}^{+}$and $\mathrm{Ni}^{2+}$ onto the surface of the powders. The reduced $\mathrm{Ni}$ can also act as the medium, which causes $\mathrm{Ni}^{2+}$ to be continuously deposited. ${ }^{16}$
Fig. 4 shows SEM images of the (a) pristine $\mathrm{CaCO}_{3}$ powders, and (b) and (c) plated powders, and (d) EDS of the plated powders. As shown in Fig. 4(a) and (b), it was found that the surface of the pristine $\mathrm{CaCO}_{3}$ powders appeared as corrugations. The rough surface of the powders could provide a large area for mechanical bonds between the powders and the plated coating, resulting in good adhesion. It was also found that welldistributed fine particles were formed on the surface of the modified $\mathrm{CaCO}_{3}$ powders after electroless plating, which resulted from the aggregation of the deposited nickel atoms to form agglomerated nickel particles, the sizes of which are all below $100 \mathrm{~nm}$. Fig. 4(d) shows the chemical compositions of the plated powders measured using EDS. The signals of nickel, phosphorous, chlorine and oxygen were found. It was suggested that Ni-P particles were deposited on the surface of the modified $\mathrm{CaCO}_{3}$ powders after electroless plating.

Fig. 5 shows a schematic diagram of electroless nickel plating onto the surface of the $\mathrm{CaCO}_{3}$ powders. As shown in Fig. 5 , the $\mathrm{CaCO}_{3}$ powders and modifying macromolecules were bound together by hydrogen bonds and intermolecular forces. On the one hand, the surface energy and the charge of the powders can be significantly decreased by adding stearic acid. This macromolecule improves the dispersion of the powders in dry conditions. Hydrophobic and hydrophilic coatings were sequentially deposited on the surface of the $\mathrm{CaCO}_{3}$ powders in that order. On the other hand, the surface of the powders was insulated by organic layers and organic-inorganic materials with core-shell structures were formed. The hydrophobic chains could effectively protect the powders from moisture and ion penetration. The surface of the powders was hydrophobic, which could prevent acid solutions (the sensitizing solution and activating solution) from touching the powders, such that $\mathrm{H}^{+}$ does not chemically react with the powders. Then, amine groups and oxhydryl groups were grafted onto the hydrophobic

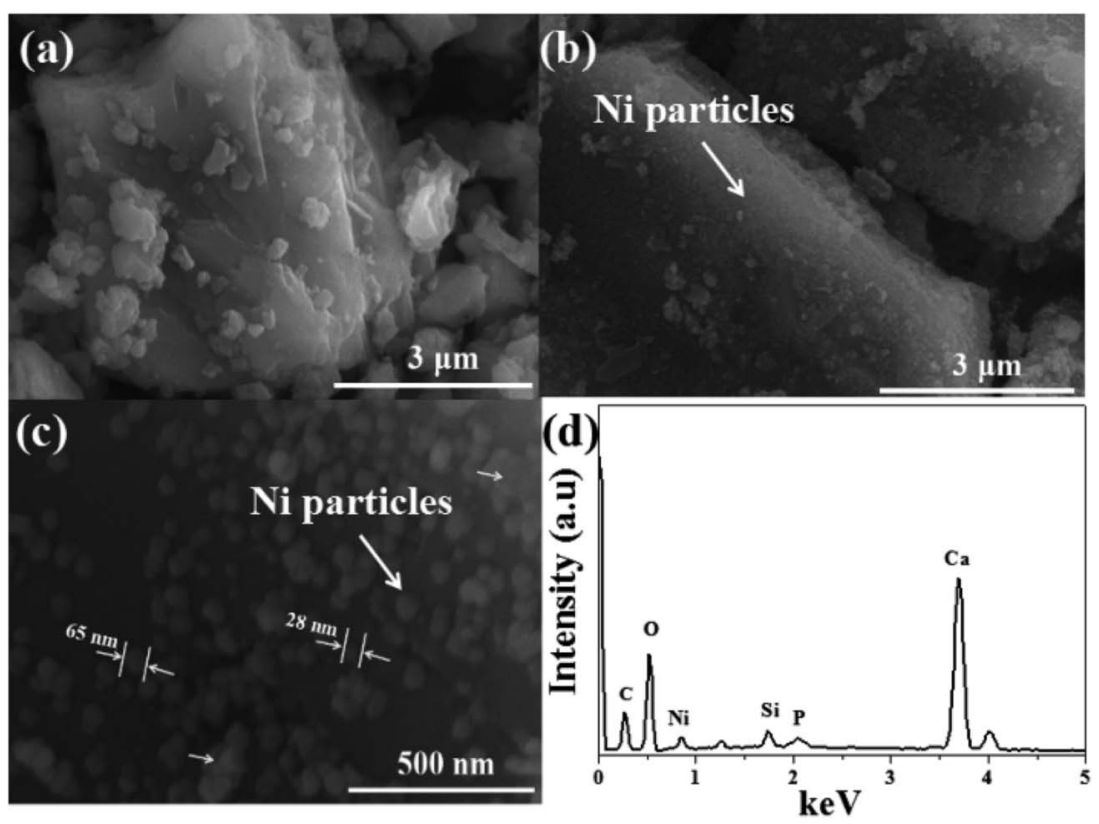

Fig. 4 SEM images of the (a) pristine $\mathrm{CaCO}_{3}$ powders, and (b) and (c) plated powders, and (d) EDS of the plated powders. 


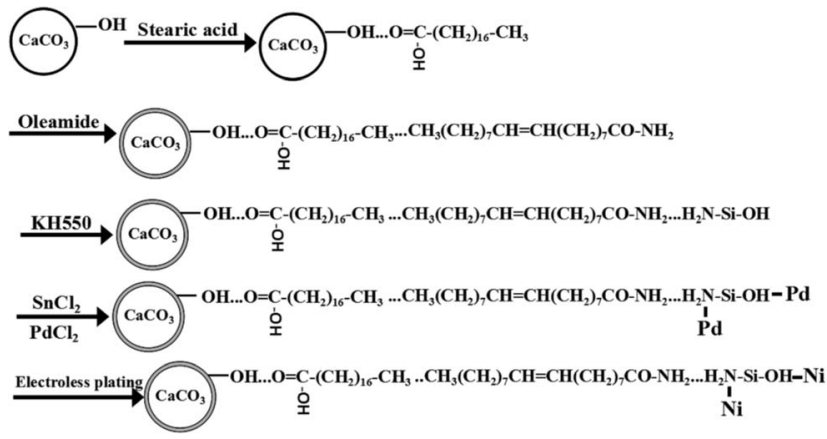

Fig. 5 Schematic diagram of the electroless nickel plating procedure.

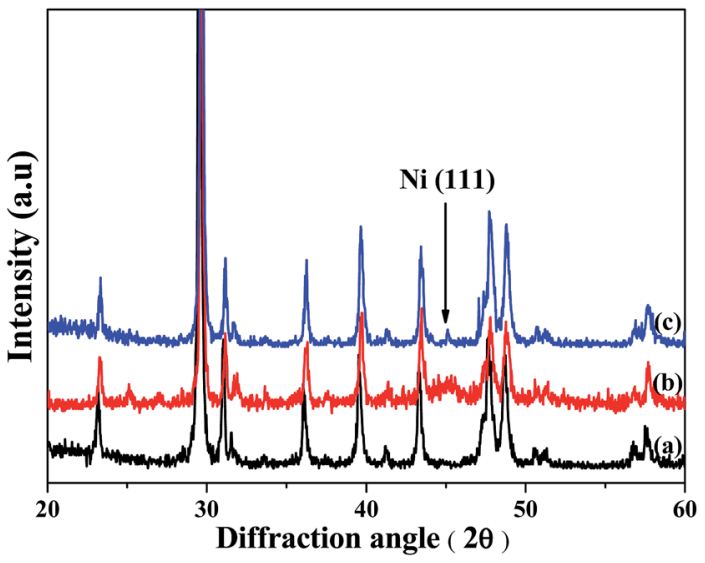

Fig. 6 XRD patterns of the (a) pristine $\mathrm{CaCO}_{3}$ powders, (b) plated powders and (c) plated powders after ultrasonic treatment.

coating when the powders were further modified with hydrolyzed KH550. At the same time, the surface of the powders became hydrophilic. The surface active groups were expected to be used as the scaffold for chemisorption of $\mathrm{Pd}^{2+}$ via their ion exchanging or coordinating behaviors. The adsorbed Pd atoms were used as a catalyst for electroless plating onto the surface of the $\mathrm{CaCO}_{3}$ powders. Ni-P particles were deposited on the surface of the modified $\mathrm{CaCO}_{3}$ powders after electroless plating.

Fig. 6 shows XRD patterns of the (a) pristine $\mathrm{CaCO}_{3}$ powders, (b) plated powders and (c) plated powders after ultrasonic treatment. The characteristic peaks at $22^{\circ}, 29^{\circ}, 35^{\circ}, 39^{\circ}, 42^{\circ}$, $47^{\circ}, 48^{\circ}$ and $57^{\circ}$ are attributed to $\mathrm{CaCO}_{3} .^{21-24}$ In addition, the peak at about $45^{\circ}$ belongs to nickel [111], as shown in Fig. 6(b). It was observed that nickel crystallites were plated on the surface of the modified $\mathrm{CaCO}_{3}$ powders. As shown in Fig. 6(c), the characteristic peak of nickel crystallites was also detected in the plated sample after ultrasonic treatment. Fig. 7 shows SEM images of the plated powders after ultrasonic treatment. It was found that nickel particles were also attached to the surface of the ultrasound-treated powders. The amine groups adsorbed Pd atoms and $\mathrm{Ni}$ atoms. The nickel particles are connected to the substrates through $\mathrm{Pd}-\mathrm{N}$ coordinate bonds. Accordingly, the fracture energy per unit area of the interface is the Pd-N bond energy, which is $2.12 \mathrm{~J} \mathrm{~m}^{-3}{ }^{23,25}$ The results indicated that the

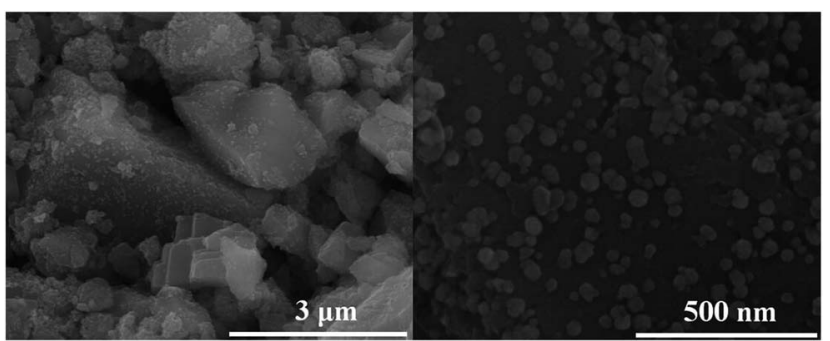

Fig. 7 SEM images of the plated powders after ultrasonic treatment.

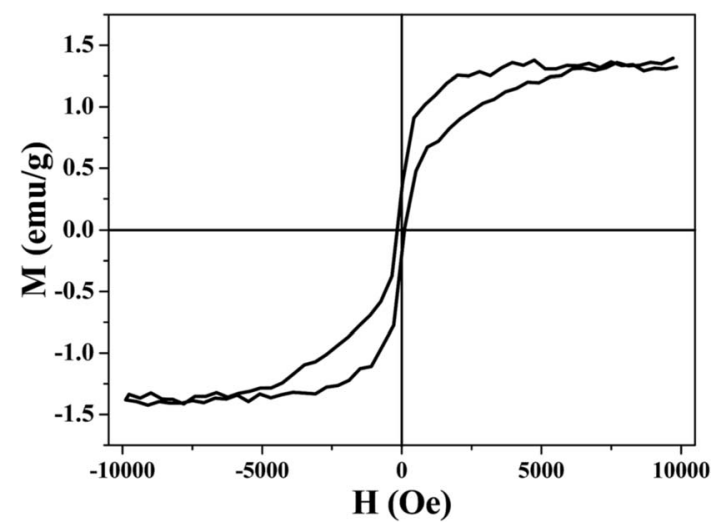

Fig. 8 Magnetic hysteresis loops of the plated $\mathrm{CaCO}_{3}$ powders.

mechanical bonds between the modified $\mathrm{CaCO}_{3}$ powders and nickel particles were strong, leading to good adhesion.

Fig. 8 shows the magnetic hysteresis loops of the plated $\mathrm{CaCO}_{3}$ powders. As shown in Fig. 8, the plated powders have a certain magnetism. The saturation magnetization of the plated $\mathrm{CaCO}_{3}$ powders was around $1.5 \mathrm{emu} \mathrm{g}^{-1}$. It is well known that $\mathrm{CaCO}_{3}$ powders are not magnetic, so the presence of magnetism suggested that nickel particles were deposited on the surface of the modified $\mathrm{CaCO}_{3}$ after electroless nickel plating, which will be useful for future applications.

\section{Conclusions}

In this work, electroless nickel plating on the surface of $\mathrm{CaCO}_{3}$ powders successively modified with stearic acid, oleamide and KH550 in that order was developed. It was found that hydrophobic chains were coated on the surface of the stearic acid and oleamide-modified powders, which could effectively protect the powders from chemical reaction with acid solutions (the sensitizing solution and activating solution). At the same time, active groups were formed on the surface of the hydrophobic coating after immersing in KH550 solution, which could adsorb palladium catalysts for electroless nickel plating. Ni-P particles were deposited on the surface of the modified $\mathrm{CaCO}_{3}$ powders after electroless plating. The size of the particles was less than $100 \mathrm{~nm}$. The mechanical bonds between the modified powders and particles were strong, leading to good adhesion. The saturation magnetization of the plated powders was around $1.5 \mathrm{emu} \mathrm{g}^{-1}$. 


\section{Acknowledgements}

The authors would like to thank the General Project of Natural Science in Colleges and Universities in Anhui Province (Grant No. KJ2015B1105905), and the Opening Project of CAS Key Laboratory of Materials for Energy Conversion (Grant No. KF2016001).

\section{References}

1 C. Li, P. Zhang and Z. Jiang, Effect of nano Cu coating on porous $\mathrm{Si}$ prepared by acid etching $\mathrm{Al}-\mathrm{Si}$ alloy powder, Electrochim. Acta, 2015, 161, 408-412.

2 J. Jiang, H. Chen, L. Zhu, et al., Effect of Heat Treatment on Structures and Mechanical Properties of Electroless Ni-PGO Composite Coatings, RSC Adv., 2016, 6, 109001-109008.

3 G. P. Ling and Y. Li, Influencing factors on the uniformity of copper coated nano- $\mathrm{Al}_{2} \mathrm{O}_{3}$ powders prepared by electroless plating, Mater. Lett., 2005, 59, 1610-1613.

4 L. Luo, Y. Wu, J. Li and Y. Zheng, Preparation of nickelcoated tungsten carbide powders by room temperature ultrasonic-assisted electroless plating, Surf. Coat. Technol., 2011, 206, 1091-1095.

5 L. Luo, J. Yu, J. Luo, et al., Preparation and characterization of Ni-coated $\mathrm{Cr}_{3} \mathrm{C}_{2}$ powder by room temperature ultrasonicassisted electroless plating, Ceram. Int., 2010, 36, 1989-1992.

6 J. P. Deepa, V. G. Resmi, T. P. D. Rajan, C. Pavithran and B. C. Pai, Studies on the influence of surface pretreatments on electroless copper coating of boron carbide particles, Appl. Surf. Sci., 2011, 257, 7466-7474.

7 G. Wen, Z. X. Guo and C. K. L. Davies, Microstructural characterisation of electroless-nickel coatings on zirconia powder, Scr. Mater., 2000, 43, 307-311.

8 A. Azarniya, F. Salatin, M. R. Eskandaripoor and A. Rasooli, A kinetic study on the mechanism of hydrogen evolution in Ni-P coated titanium hydride powder, Adv. Powder Technol., 2014, 26, 259-266.

9 Z. J. Song, J. L. Xie, P. H. Zhou, X. H. Wang, T. H. Liu and L. J. Deng, Toughened polymer composites with flake carbonyl iron powders and their electromagnetic/ absorption properties, J. Alloys Compd., 2013, 551, 677-681.

10 S. Y. Tong, M. J. Tung, W. S. Ko, Y. T. Huang, Y. P. Wang, L. C. Wang and J. M. Wu, Effect of Ni fillers on microwave absorption and effective permeability of NiCuZn ferrite/Ni/ polymer functional composites, J. Alloys Compd., 2013, 550, 39.

11 Z. P. Sun, J. J. Huang, L. B. Wang, X. C. Zhang, M. L. Li and B. Tang, Method for electroless nickel plating on poly(ethylene terephthalate) substrate modified with primer and self-assembled monolayer, J. Mater. Sci.: Mater. Electron., 2015, 26, 10132-10137.
12 I. Lee, P. T. H. And and M. F. Rubner, Selective Electroless Nickel Plating of Particle Arrays on Polyelectrolyte Multilayers, Chem. Mater., 2003, 15, 4583-4589.

13 Y. Liu, Y. Xu, Y. Zhu, Y. Liu, S. Zheng, Y. Liu, A. Langrock and C. Wang, Selenium@mesoporous carbon composite with superior lithium and sodium storage capacity, ACS Nano, 2013, 7, 3627-3634.

14 C. Viravaidya, M. Li and S. Mann, Microemulsion-based synthesis of stacked calcium carbonate (calcite) superstructures, Chem. Commun., 2004, 10, 2182.

15 R. Herrera, X. Erdocia, R. Llano-Ponte and J. Labidi, Characterization of hydrothermally treated wood in relation to changes on its chemical composition and physical properties, J. Anal. Appl. Pyrolysis, 2014, 107, 256266.

16 Q. Zhou, H. Chen and Y. Wang, Region-selective electroless gold plating on polycarbonate sheets by UV-patterning in combination with silver activating, Electrochim. Acta, 2010, 55, 2542-2549.

17 J. L. Cao, Z. K. Wua, J. Yang, S. Y. Lia, H. X. Tang and G. Y. Xie, Site-selective electroless plating of copper on a poly(ethylene terephthalate) surface modified with a selfassembled monolayer, Colloids Surf., A, 2012, 415, 374-379.

18 J. Y. Choi, T. J. Kim, S. Lee, et al., Drop-on-demand printing of conductive ink by electrostatic field induced inkjet head, Appl. Phys. Lett., 2008, 93, 193508.

19 V. Dimitrov and T. Komatsu, Classification of Simple Oxides: A Polarizability Approach, J. Solid State Chem., 2002, 163, 100-112.

20 S. L. Ma, B. Xu, G. Z. Wu, Y. F. Wang, F. Ma, D. Y. Ma, K. W. Xu and T. Bell, Microstructure and mechanical properties of SiCN hard films deposited by an arc enhanced magnetic sputtering hybrid system, Surf. Coat. Technol., 2008, 202, 5379-5382.

21 Z. P. Sun, J. J. Huang, Q. Liu, M. Gao, M. Y. Li, F. Zhao, W. Cheng and B. Tang, Effects of temperature on $\mathrm{Ni}$ coating on poly(ethylene terephthalate) substrate modified with primer, J. Mater. Sci.: Mater. Electron., 2016, 27, 58925898.

22 R. L. Blaine and H. E. Kissinger, Homer Kissinger and the Kissinger equation, Thermochim. Acta, 2012, 14, 1-6.

23 M. L. Wang, Z. G. Yang, C. Zhang, et al., Trans. Mater. Heat Treat., 2015, 7, 183-189.

24 J. Georgieva and S. Armyanov, Electroless deposition and some properties of $\mathrm{Ni}-\mathrm{Cu}-\mathrm{P}$ and Ni-Sn-P coatings, J. Solid State Electrochem., 2007, 11, 869-876.

$25 \mathrm{H}$. E. Evans, Stress effects in high temperature oxidation of metals, Int. Mater. Rev., 1995, 40, 1-40. 